\title{
Características de los pacientes infectados por el coronavirus 2019 en China y dinámica de su transmisión temprana
}

\author{
Characteristics of patients infected with coronavirus 2019 in China and dynamics of its early transmission
}

\section{Comentado de:}

Huang C, et al. Lancet 2020; published online Jan 24. PMID: $32007144^{1}$ y Li Q, et al. [published online ahead of print, 2020 Jan 29]. N Engl J Med. 2020;10.1056/NEJMoa2001316. PMID: $319958573^{2}$.

\section{Características clínicas de los pacientes infectados por el nuevo coronavirus 2019 en Wuhan, China \\ Huang C, et al. Lancet 2020; published online Jan 24.}

\section{Antecedentes}

Ante la reciente aparición de casos de neumonía en Wuhan, China, causados por un nuevo betacoronavirus, denominado coronavirus 2019 (2019-nCoV), en este artículo se reportan las características epidemiológicas, clínicas, de laboratorio y radiológicas, así como el tratamiento instaurado y los resultados clínicos de los primeros pacientes infectados.

\section{Métodos}

Todos los pacientes con sospecha de infección por el nuevo coronavirus, 2019-nCoV, fueron ingresados en un hospital designado en Wuhan, China. Se recopilaron y analizaron prospectivamente datos sobre pacientes con infección por 2019-nCoV confirmada por laboratorio mediante reacción en cadena de la polimerasa con transcriptasa inversa (RT-PCR, por sus iniciales en inglés) en tiempo real y secuenciación de próxima generación. Los datos se recolectaron mediante formularios estandarizados, compartidos por el Consorcio Internacional de Infecciones Respiratorias Agudas y Emergentes de registros médicos electrónicos. Los investigadores también se comunicaron en forma directa con

\section{Dinámica de transmisión temprana en Wuhan, China,} de la neumonía infectada por el nuevo coronavirus

Li Q, et al. [published online ahead of print, 2020 Jan 29]. N Engl J Med. 2020;10.1056/NEJMoa2001316

\section{Antecedentes}

Los casos iniciales de neumonía por 2019-nCoV se detectaron en Wuhan (provincia de Hubei, China), en diciembre de 2019 y enero de 2020. Se analizaron los datos de los primeros 425 casos confirmados en Wuhan para determinar las características epidemiológicas de la neumonía por 2019-nCov (NPC).

\section{Métodos}

Se recopiló información sobre las características demográficas, el historial de exposición y la línea de tiempo de la enfermedad de los casos confirmados por laboratorio de NPC e informados antes del 22 de enero de 2020. Se describieron las características de los casos y se estimaron las distribuciones epidemiológicas clave de retrasos de tiempo (variables necesarias para que los modelos matemáticos que simulan la dinámica de las enfermedades infectocontagiosas se comporten de manera más parecida al proceso real). En el período inicial de crecimiento exponencial se estimó el tiempo de duplicación de la epidemia y el número reproductivo básico (este es el número promedio de casos nuevos que genera un caso dado a lo largo de un período infeccioso, y permite estimar la velocidad con que una enfermedad puede propagarse en los pacientes o sus familias para documentar los datos epidemiológicos y los síntomas. Los resultados también se compararon entre los pacientes que habían ingresado en la unidad de cuidados intensivos ( $\mathrm{UCl})$ y los que no lo habían hecho.

\section{Recomendaciones}

Para el 2 de enero de 2020, 41 pacientes admitidos en el hospital habían sido identificados por el laboratorio como infectados por el 2019-nCoV (ver Tabla 1). Todos los pacientes tenían neumonía con hallazgos anormales en la tomografía computada de tórax. Las complicaciones incluyeron: síndrome de dificultad respiratoria aguda (12 pacientes, $29 \%$ ), ARNaemia (resultado positivo para RT-PCR en tiempo real en la muestra de plasma) (6 pacientes, $15 \%$ ), daño cardíaco agudo (5 pacientes, $12 \%$ ) e infección secundaria (4 pacientes, $10 \%)$. Unos 13 pacientes (32\%) fueron ingresados en una $\mathrm{UCl}$, mientras que $6(15 \%)$, fallecieron.

\section{Interpretación}

La infección por 2019-nCoV ocasionó enfermedad respiratoria grave similar al coronavirus del síndrome respiratorio agudo severo (SARS-Cov), y se asoció con mayor admisión a la UCI y una alta mortalidad. Para superar las brechas en el conocimiento sobre el origen, la epidemiología, la duración de la transmisión humana y el espectro clínico de la enfermedad deberán realizarse estudios adicionales.

\section{Fuente de financiamiento}

Ministerio de Ciencia y Tecnología, Academia China de Ciencias Médicas, Fundación Nacional de Ciencias Naturales de China y Comisión Municipal de Ciencia y Tecnología de Beijing.

\section{una población).}

\section{Resultados}

Entre los primeros 425 pacientes con NPC confirmada, la edad promedio fue 59 años y $56 \%$, de sexo masculino. La mayoría (55\%) de los casos con inicio antes del 1 de enero de 2020, estaban vinculados al mercado mayorista de mariscos de Huanan, en comparación con el 8,6\% de los casos posteriores. El período de incubación promedio fue 5,2 días (intervalo de confianza [IC] del 95\%: 4,1 a 7,0), con percentilo 95 de la distribución a los 12,5 días. En sus primeras etapas, la epidemia duplicó su tamaño cada 7,4 días. Con un intervalo serial promedio de 7,5 días (IC $95 \% 5,3$ a 19), el número reproductivo básico se estimó en 2,2 (IC $95 \%$ 1,4 a 3,9).

\section{Conclusiones}

Sobre la base de esta información, existe evidencia de que se ha producido una transmisión de persona a persona del 2019 $\mathrm{nCoV}$ entre los contactos cercanos desde mediados de diciem bre de 2019. Se requerirán esfuerzos considerables para reducir la transmisión y para controlar los brotes si se producen dinámicas similares en otros ámbitos. Deberían implementarse medidas para prevenir o reducir la transmisión de la infección en poblaciones en riesgo.

\section{Fuente de financiamiento}

Ministerio de Ciencia y Tecnología de China y otros. 
Tabla 1. Características de los pacientes infectados, comparación entre los ingresados a la Unidad de Cuidados Intensivos con los no ingresados. Fuente: Huang C, y col. Lancet 2020; published online Jan 24. Notas: UCl = unidad de cuidados intensivos. Los datos corresponden a la mediana (RIC), $\mathrm{n}(\%) / \mathrm{N}(\%)$, donde $\mathrm{N}$ es el número total de pacientes con datos disponibles. Los valores de $\mathrm{p}$ que comparan la admisión en la $\mathrm{UCI}$ y la no admisión en la UCI fueron realizados con la prueba exacta de Fisher o la prueba U de Mann-Whitney.

\begin{tabular}{|c|c|c|c|c|}
\hline Características & Total de pacientes $(n=41)$ & Ingresados a UCI* $(n=13)$ & No UCl* $(n=28)$ & P-valor \\
\hline Edad, años & $49,0(41,0$ a 58,0$)$ & $49,0(41,0$ a 61,0$)$ & $49,0(41,0$ a 57,5$)$ & 0,60 \\
\hline Sexo masculino & $30(73 \%)$ & $11(85 \%)$ & $19(68 \%)$ & 0,24 \\
\hline $\begin{array}{l}\text { Exposición al mercado de maris- } \\
\text { cos }\end{array}$ & $27(66 \%)$ & $9(69 \%)$ & $18(64 \%)$ & 0,75 \\
\hline Signos y síntomas & & & & \\
\hline Fiebre & $40(98 \%)$ & $13(100 \%)$ & 27 (96 \%) & 0,68 \\
\hline Tos & $31(76 \%)$ & $11(85 \%)$ & $20(71 \%)$ & 0,35 \\
\hline Mialgia o fatiga & $18(44 \%)$ & $7(54 \%)$ & $11(39 \%)$ & 0,38 \\
\hline Cefalea & $3 / 38(8 \%)$ & 0 & $3 / 25(12 \%)$ & 0,10 \\
\hline Hemoptisis & $2 / 39(5 \%)$ & $1(8 \%)$ & $1 / 26(4 \%)$ & 0,46 \\
\hline Diarrea & $1 / 38(3 \%)$ & 0 & $1 / 25(4 \%)$ & 0,66 \\
\hline Disnea & $22 / 40(55 \%)$ & $12(92 \%)$ & $10 / 27(37 \%)$ & 0,0010 \\
\hline $\begin{array}{l}\text { Días desde el inicio de la enfer- } \\
\text { medad hasta la disnea }\end{array}$ & $8,0(5,0$ a 13,0$)$ & $8,0(6,0$ a 17,0$)$ & $6,5(2,0$ a 10,0$)$ & 0,22 \\
\hline $\begin{array}{l}\text { Días desde la primera admisión } \\
\text { hasta el traslado }\end{array}$ & $5,0(1,0$ a 8,0$)$ & $8,0(5,0$ a 14,0$)$ & $1,0(1,0$ a 6,5$)$ & 0,002 \\
\hline Presión sistólica, mm Hg & $125,0(119,0$ a 135,0$)$ & $145,0(123,0$ a 167,0$)$ & $122,0(118,5$ a 129,5$)$ & 0,018 \\
\hline
\end{tabular}

\section{Comentario}

El 31 de diciembre de 2019 se comunicaron a la Organización Mundial de la Salud (OMS) varios casos de neumonía en Wuhan, China. Se trataba de un virus distinto a los conocidos, lo cual resultó preocupante porque no se sabía de qué manera este nuevo virus podía afectar a las personas. Los artículos resumidos son parte de los primeros reportes acerca de las características de la infección por el 2019-nCoV ${ }^{1,2}$.

Con el correr de los días y ante la creciente magnitud del brote, la OMS declaró la emergencia de salud pública de importancia internacional ${ }^{3}$.

Hasta el día 5 de febrero del corriente año se han reportado 24.554 casos confirmados, de los cuales 24.363 fueron en China. Los casos confirmados hasta el momento se localizaron en 24 países, y se produjeron 492 muertes ${ }^{4}$.

Se presume que la fuente del brote es zoonótica, aunque el reservorio animal/huésped intermedio no ha sido identificado 5 . Tal como lo describe el artículo de Li et al., se detectó transmisión de humano a humano con un período de incubación estimado entre 2 y 10 días (estas aproximaciones se refinarán a medida que haya más datos disponibles) ${ }^{2}$.

La infección puede ocasionar enfermedad severa y muerte, con un espectro clínico completo que todavía queda pendiente determinar. La tasa de letalidad reportada se encuentra alrededor del $2 \%$ (menor a la causada por el SARS-Cov, que fue cercana al $10 \%)^{5}$.

No existe un tratamiento antiviral específico recomendado para la infección por 2019-nCoV. Las personas infectadas reciben tratamiento de sostén. 
Para los países sin casos, como Argentina, la OMS sugiere: a) asegurar la vigilancia para detectar eventos respiratorios agudos; b) garantizar que los trabajadores de la salud tengan acceso a información actualizada sobre esta enfermedad; c) verificar las prácticas de prevención y control de infecciones en los establecimientos de salud, d) que el personal de salud esté familiarizados con los principios y procedimientos para manejar las infecciones por el 2019-nCoV y esté capacitado para consultar sobre el historial de viajes de un paciente para vincular esta información con datos clínicos ${ }^{5}$. Con este fin, el Ministerio de Salud de la Nación de Argentina ha emitido una serie de recomendaciones para el equipo de salud ${ }^{6}$.

\section{Conclusiones de la comentadora}

Estamos ante el inicio de un brote causado por un nuevo virus del cual todavía se desconocen muchos aspectos. Algunas de sus características se empiezan a esclarecer a partir de estudios como estos, y en los próximos meses seguramente se contará con más información. Aún en los países en los que no se han registrado casos, es importante que los profesionales de la salud se mantengan alerta y estén informados.

Carolina Carrara [ Servicio de Medicina Familiar y Comunitaria, Hospital Italiano de Buenos Aires, CABA, Ciudad Autónoma de Buenos Aires, Argentina. carolina.carrara@hospitalitaliano.org.ar ]

Carrara C. Características de los pacientes infectados por el coronavirus 2019 en China y dinámica de su transmisión temprana. Evid Actual Pract Ambul. 2020;23(1):e002040. Comentado de: Huang C, et al. Clinical features of patients infected with 2019 novel coronavirus in Wuhan. Lancet 2020; published online Jan 24. PMID: 32007144; y Li Q, et al. Early Transmission Dynamics in Wuhan, China, of Novel Coronavirus-Infected Pneumonia. [published online ahead of print, 2020 Jan 29]. N Engl J Med. 2020;10.1056/NEJMoa2001316. PMID: 319958573

\section{Referencias}

1. Huang C, Wang Y, Li X, et al. Clinical features of patients infected with 2019 novel coronavirus in Wuhan. Lancet. 2020;(20):30183-30188.

2. Li Q, Guan X, Wu P, Wang X, et al. Early Transmission Dynamics in Wuhan, China, of Novel Coronavirus-Infected Pneumonia. N Engl J Med. 2020;

3. Statement on the second meeting of the International Health Regulations (2005) Emergency Committee regarding the outbreak of novel coronavirus (2019-nCoV); 2020. Available from: https://www.who.int/news-room/detail/30-01-2020-statement-on-the-second-meeting-of-the-internationalhealth-regulations-(2005)-emergency-committee-regarding-the-outbreak-of-novel-coronavirus-(2019-ncov [Last access: 2020-02-03].

4. Novel Coronavirus(2019-nCoV) Situation Report - 16; 2020. Available from: https://www.who.int/docs/default-source/coronaviruse/situation-reports/ 20200203-sitrep-14-ncov.pdf?sfvrsn=f7347413_2 [Last access: 2020-02-06].

5. Novel Coronavirus (2019-nCoV) technical guidance; 2020. Available from: https://www.who.int/emergencies/diseases/novel-coronavirus-2019/ technical-guidance [Last access: 2020-02-03].

6. Recomendaciones para equipos de salud. Nuevo coronavirus 2019-nCoV; 2020. Available from: https://www.argentina.gob.ar/salud/ recomendaciones-equipos-de-salud-coronavirus-2019-ncov [Last access: 2020-02-03]. 